\title{
Tissue source determines the differentiation potentials of mesenchymal stem cells: a comparative study of human mesenchymal stem cells from bone marrow and adipose tissue
}

Liangliang X $\mathrm{u}^{1,3+}$, Yamei Liu ${ }^{2+}$, Yuxin Sun ${ }^{3}$, Bin Wang ${ }^{3}$, Yunpu Xiong ${ }^{7}$, Weiping Lin ${ }^{3}$, Qiushi Wei ${ }^{1}$, Haibin Wang ${ }^{1}$, Wei He ${ }^{1,9^{*}}$, Bin Wang ${ }^{7^{*}}$ and Gang Li $i^{3,4,5,6,8^{*}}$

\begin{abstract}
Background: Mesenchymal stem cells (MSCs) possess intrinsic regeneration capacity as part of the repair process in response to injury, such as fracture or other tissue injury. Bone marrow and adipose tissue are the major sources of MSCs. However, which cell type is more effective and suitable for cell therapy remains to be answered. The intrinsic molecular mechanism supporting the assertion has also been lacking.

Methods: Human bone marrow-derived MSCs (BMSCS) and adipose tissue-derived MSCs (ATSCs) were isolated from bone marrow and adipose tissue obtained after total hip arthroplasty. ATSCs and BMSCs were incubated in standard growth medium. Trilineage differentiation including osteogenesis, adipogenesis, and chondrogenesis was performed by addition of relevant induction mediums. The expression levels of trilineage differentiation marker genes were evaluated by quantitative RT-PCR. The methylation status of CpG sites of Runx2, PPARY, and Sox9 promoters were checked by bisulfite sequencing. In addition, ectopic bone formation and calvarial bone critical defect models were used to evaluate the bone regeneration ability of ATSCs and BMSCs in vivo.

Results: The results showed that BMSCs possessed stronger osteogenic and lower adipogenic differentiation potentials compared to ATSCs. There was no significant difference in the chondrogenic differentiation potential. The CpG sites of Runx2 promoter in BMSCs were hypomethylated, while in ATSCs they were hypermethylated. The CPG sites of PPARY promoter in ATSCs were hypomethylated, while in BMSCs they were hypermethylated. The methylation status of Sox9 promoter in BMSCs was only slightly lower than that in ATSCs.

(Continued on next page)
\end{abstract}

\footnotetext{
*Correspondence: hewei1123@gzucm.edu.cn; wangbin1973@163.com; gangli@cuhk.edu.hk

${ }^{\dagger}$ Equal contributors

'Key Laboratory of Orthopaedics \& Traumatology, The First Affiliated Hospital of Guangzhou University of Chinese Medicine, The First Clinical Medical College, Guangzhou University of Chinese Medicine, Guangzhou, China

${ }^{7}$ Department of Traumatology, The Third Affiliated Hospital of Guangzhou University of Traditional Chinese Medicine, Guangzhou, Guangdong 510240, People's Republic of China

${ }^{3}$ Department of Orthopaedics \& Traumatology, Faculty of Medicine, The Chinese University of Hong Kong, Prince of Wales Hospital, Shatin, Hong Kong, Special Administrative Region of China

Full list of author information is available at the end of the article
} 
(Continued from previous page)

Conclusions: The epigenetic memory obtained from either bone marrow or adipose tissue favored MSC differentiation along an osteoblastic or adipocytic lineage. The methylation status of the main transcription factors controlling MSC fate contributes to the differential differentiation capacities of different source-derived MSCs.

Keywords: Mesenchymal stem cells, Epigenetic regulation, Bone marrow-derived MSCs, Adipose tissue-derived MSCs

\section{Background}

Mesenchymal stem cells (MSCs) possess intrinsic regeneration capacity as part of the repair process in response to injury, such as fracture or other tissue injury. Several characteristics of MSCs, such as the potential to differentiate into multiple lineages and the ability to be easily expanded ex vivo while retaining their original lineage differentiation commitment, make these cells very promising targets for therapeutic use in regenerative medicine and tissue engineering [1]. However, low cell survival rate and differentiation capacity in vivo after MSC transplantation have significantly reduced the effectiveness of stem cell therapy [2-5]. Over the past decade, MSCs have been isolated from the umbilical cord, umbilical cord blood, bone marrow, adipose tissue, and many other adult tissues. To date, bone marrow-derived MSCs (BMSCs) and adipose tissue-derived MSCs (ATSCs) are still the main source of MSCs, especially in autologous cell-based therapies, due to ease of harvest and potential autologous application [6]. An important question, which type of cell is more effective and suitable for cell therapy remains unknown. Many studies have shown that BMSCs and ATSCs share similar features, including the morphology and cell surface markers, but significant biologic differences have been found concerning their proliferation and differentiation capacities [7-10]. Conflicting results have been reported; some studies indicate that the clinical application potential of ATSCs is more effective than or as effective as that of BMSCs, while other studies conclude that BMSCs are superior to ATSCs [11-13]. In addition, significant differences in the cytokine secretome and chemokine receptor expression between ATSCs and BMSCs have also been reported [14, 15]. Despite different gene expression and differentiation capacities have been observed among ATSCs and BMSCs, the underlying mechanisms regarding epigenetic regulation are yet to be investigated.

Interestingly, recent studies from both our group and others have demonstrated that epigenetic regulation is an important factor to control MSC differentiation and proliferation [16]. Up to now, DNA methylation and histone modifications are the most important epigenetic regulations which possess the power to control the differentiation or maintain the self-renewal of MSCs [17]. Changes in the methylation states of the $\mathrm{CpG}$ islands in the promoter regions or the first exon are known to be inversely responsible for expression of the corresponding genes. The bivalent loci in MSCs are often low in DNA methylation and can be further methylated or activated, which are distinct from those in the embryonic stem cells and differentiated cells [18]. Targeted DNA methylation within the Trip10 promoter has been shown to accelerate the MSCs to neuron or osteocyte differentiation [19].

In the present study, we determined the effect of epigenic regulation of MSC fate. The results showed that epigenetic memory obtained from either bone marrow or adipose tissue favored their differentiation along an osteoblastic or adipocytic lineage. The $\mathrm{CpG}$ sites of Runx2 promoter in BMSCs were hypomethylated, while in ATSCs they were hypermethylated. The CpG sites of PPAR $\gamma$ promoter in ATSCs were hypomethylated, while in BMSCs they were hypermethylated. The methylation status of Sox9 promoter in BMSCs was only slightly lower than that in ATSCs. We concluded that the methylation status of the main transcription factors controlling MSC fate contributed to the differential differentiation capacities of different source-derived MSCs.

\section{Methods}

Isolation and culture of human BMSCs and ATSCs

ATSCs and BMSCs were prepared as described previously $[20,21]$. Briefly, the BMSCs were fractionated on a Ficoll density gradient (Ficoll-Paque ${ }^{\mathrm{Tm}}-\mathrm{PLUS}$; Amersham Pharmacia, Sweden) and the MSCenriched fraction was washed, seeded in culture flasks, and maintained at $37{ }^{\circ} \mathrm{C}$ in a humidified atmosphere. The adipose tissue was washed extensively with equal volumes of phosphate-buffered saline (PBS), and the extracellular matrix was digested with 0.075\% collagenase (type I; Sigma-Aldrich, St Louis, MO, USA) at $37{ }^{\circ} \mathrm{C}$ for $30 \mathrm{~min}$. With $\alpha$-MEM containing $10 \% \mathrm{FBS}$ and antibiotics $(100 \mathrm{U} / \mathrm{ml}$ penicillin $\mathrm{G}$ and $100 \mu \mathrm{g} / \mathrm{ml}$ streptomycin), the sample was centrifuged at $1200 \times g$ for $10 \mathrm{~min}$. The cell pellet was resuspended in $160 \mathrm{mM} \mathrm{NH} \mathrm{NH}_{4} \mathrm{Cl}$ and incubated at room temperature for $10 \mathrm{~min}$. After removing cellular remains through a $100-\mu \mathrm{m}$ Nylon mesh (Cell Strainer; Becton Dickinson and Company, Franklin Lakes, NJ, USA), the cells were incubated in the culture medium. The adhered ATSCs were cultured for about 
2 weeks, and nearly all cells transformed into fibroblast-like cells, which are morphologically similar to BMSCs.

\section{Phenotypic characterization of hMSCs}

After reaching $80 \%$ confluence, the cells were rinsed twice with PBS and treated with $0.05 \%$ trypsin-EDTA for $2 \mathrm{~min}$. Serum-containing medium was then immediately added to the culture to end trypsinization. The fluid was then collected and centrifuged $(800 \times g$ for $5 \mathrm{~min}$ ). After discarding the supernatant, the precipitate was resuspended in staining buffer and incubated with fluorochrome-conjugated primary antibodies against CD34, CD44, CD45, CD73, CD90, and CD105 or corresponding isotype control (BD Biosciences, USA) at $4{ }^{\circ} \mathrm{C}$ for $30 \mathrm{~min}$. The stained cells were immediately detected using flow cytometry (BD Biosciences, USA).

\section{Osteogenic differentiation}

MSCs were plated at $4 \times 10^{3}$ cells $/ \mathrm{cm}^{2}$ in a 12 -well plate and cultured in the basal medium until the cells reached confluence. The cells were then incubated in osteogenic induction medium (OIM), which is basal medium supplemented with $1 \mathrm{nM}$ dexamethasone, $50 \mu \mathrm{M}$ ascorbic acid, and $20 \mathrm{mM} \beta$-glycerolphosphate (all from SigmaAldrich), at $37{ }^{\circ} \mathrm{C}, 5 \% \mathrm{CO}_{2}$ as described previously [20, 21]. At day 14, the mineralization of MSCs was assessed by Alizarin Red S staining. Briefly, to evaluate the mineralized nodule formation in vitro, the cell/matrix layer was washed with PBS, fixed with $70 \%$ ethanol for 10 min, and stained with $0.5 \%$ Alizarin Red S (pH 4.1; Sigma, St Louis, MO, USA) for $5 \mathrm{~min}$.

\section{Adipogenic differentiation}

MSCs were plated at $4 \times 10^{3}$ cells $/ \mathrm{cm}^{2}$ in a six-well culture plate and cultured until the cells reached confluence. The medium was then replaced with adipogenic induction medium (AIM), which is basal medium supplemented with $500 \mathrm{nM}$ dexamethasone, $0.5 \mathrm{mM}$ isobutylmethylxanthine, $50 \mathrm{mM}$ indomethacin, and $10 \mathrm{mg} / \mathrm{ml}$ of insulin (all from Sigma-Aldrich). The cells were cultured for another 21 days, and then the cells were fixed with $70 \%$ ethanol for $10 \mathrm{~min}$ and stained with $0.3 \%$ fresh Oil Red O solution (Sigma-Aldrich) for $10 \mathrm{~min}$. The wells were rinsed three times with distilled water and viewed using a LEICA Q500MC microscope (Leica Cambridge Ltd).

\section{Chondrogenic differentiation}

For chondrogenic differentiation, a micromass culture system was used. MSCs (in $5 \mu \mathrm{l}$ ) at a centration of $1.6 \times$ $10^{7}$ cells $/ \mathrm{ml}$ were dropped in the centers of 24-well plates. The plates were placed in incubator at $37{ }^{\circ} \mathrm{C}, 5 \%$ $\mathrm{CO}_{2}$ without culture medium for 2 hours. These cells were then cultured in chondrogenic induction medium (CIM), which is basal medium supplemented with $10 \mathrm{ng} /$ $\mathrm{ml}$ transforming growth factor- $\beta 3$ (R\&D Systems), $500 \mathrm{ng} /$ $\mathrm{ml}$ bone morphogenetic protein-2 (R\&D Systems), $10^{-7} \mathrm{M}$ dexamethasone, $50 \mathrm{mg} / \mathrm{ml}$ ascorbate-2-phosphate, $40 \mathrm{mg} /$ $\mathrm{ml}$ proline, $100 \mathrm{mg} / \mathrm{ml}$ pyruvate (all from Sigma-Aldrich), and 1:100 diluted ITS + Premix $(6.25 \mathrm{mg} / \mathrm{ml}$ insulin, $6.25 \mathrm{mg} / \mathrm{ml}$ transferrin, $6.25 \mathrm{mg} / \mathrm{ml}$ selenous acid, $1.25 \mathrm{mg} / \mathrm{ml}$ bovine serum albumin, and $5.35 \mathrm{mg} / \mathrm{ml} \mathrm{lino-}$ leic acid) (Becton Dickinson). The chondrogenic medium was changed every 3 days.

\section{Quantitative real-time RT-PCR}

The cells were harvested and homogenized for RNA extraction with the RNeasy mini kit (Qiagen, Hilden, Germany). The mRNA was reverse-transcribed to cDNA by the PrimeScript First Strand cDNA Synthesis Kit (TaKaRa). Then $5 \mu \mathrm{l}$ of total cDNA from each sample was amplified in a final volume of $25 \mu \mathrm{l}$ of reaction mixture containing Platinum SYBR Green, qPCR SuperMix-UDG ready-to-use reaction cocktail, and specific primers using the ABI StepOne Plus system (all from Applied Biosystems, CA, USA). The expression level of the target gene was normalized to that of the $\beta$-actin gene, which was shown to be stable in this study. Relative gene expression was calculated with the $2^{-\triangle \mathrm{CT}}$ formula. The sequences of the primers were presented in Additional file 1: Table S1.

\section{DNA isolation and bisulfite treatment}

Genomic DNA was isolated from MSCs using the PureLink $^{\circ}$ Genomic DNA isolation kit following the manufacturer's instructions (Invitrogen). Bisulfite modification was done as described previously [22]. Briefly, about $2 \mu \mathrm{g}$ of genomic DNA was denatured by $\mathrm{NaOH}$ (final concentration $0.2 \mathrm{~mol} / \mathrm{L}$ ) for $10 \mathrm{~min}$ at $37{ }^{\circ} \mathrm{C}$. Hydroquinone and sodium hydroxide were added, and samples were incubated at $50{ }^{\circ} \mathrm{C}$ for 16 hours. Modified DNA was purified using the Wizard DNA Clean-Up System following the manufacturer's instructions (Promega) and eluted into $50 \mu \mathrm{l}$ of water. DNA was treated with $\mathrm{NaOH}$ (final concentration $0.3 \mathrm{~mol} / \mathrm{l}$ ) for $5 \mathrm{~min}$ at room temperature, ethanol precipitated, and resuspended in $20 \mu \mathrm{l}$ of water. Modified DNA was used immediately or stored at $-20{ }^{\circ} \mathrm{C}$.

\section{Bisulfite sequencing}

Bisulfite-modified genomic DNA was amplified by PCR. All PCRs were carried out using $\mathrm{KAPA}^{2 \mathrm{G}^{\mathrm{m}}}$ Fast HotStart DNA Polymerase Polymerase. The sequences of primers used for the bisulfite sequencing analysis are presented in Additional file 1: Table S2. PCR products were run on $1.5 \%$ agarose gels and bands were excised using the TaKaRa MiniBEST Agarose Gel DNA Extraction Kit following the manufacturer's instructions 
(TaKaRa). Purified bands were cloned using the $\mathrm{pMD}^{\mathrm{mm}} 19-\mathrm{T}$ Vector Cloning Kit following the manufacturer's instructions (TaKaRa). Colonies were selected and grown overnight in Luria-Bertani medium containing ampicillin $(100 \mu \mathrm{g} / \mathrm{ml})$ with shaking at $37{ }^{\circ} \mathrm{C}$. Plasmid DNA was isolated using the TaKaRa MiniBEST Agarose Gel DNA Extraction Kit following the manufacturer's instructions (TaKaRa). Plasmids were sequenced using the M13 universal reverse primer (BGI).

\section{Ectopic bone formation}

In-vivo studies were performed with the approval of the Animal Experimentation Ethics Committee of The Chinese University of Hong Kong. After anesthesia, an incision was made on the dorsum and a subcutaneous pocket was created. $2.5 \times 10^{6}$ ATSCs or BMSCs were seeded onto sterilized Skelite ${ }^{\circ}$ resorbable Si-TCP bone graft substitute, and $\mathrm{Si} / \mathrm{TCP}$ cubes with PBS served as the control group. The cells were then transplanted into the same mice. The wound was then closed in layers. At week 8, the scaffolds with cells were harvested for HE staining, as well as immohistochemical staining of osteocalcin (OCN). The osteoid matrix areas were measured using ImageJ software, and five microscopic fields were chosen from each sample and measured.

\section{Calvarial bone critical defect model}

Six nude mice (6 weeks old, body weight $50 \mathrm{~g}$ ) were used. All animals were placed under general anesthesia with a dosage of $0.2 \mathrm{ml} / 100 \mathrm{~g}$ body weight via intraperitoneal injection of a combination of ketamine, xylazine, and saline at a ratio of 3:2:3. The dorsal part of animal's cranium was shaved and disinfected with iodine solution. The skin and underlying tissues including the periosteum were detached to expose the parietal bones on both sides. One piece of circular bone was removed in the middle region of the cranium using a hollow trephine bur with a 5$\mathrm{mm}$ outer diameter. Continuous irrigation with sterile PBS was used to prevent overheating of the bone margins and to maintain moisture in the tissue. Any animal with evidence of meninges injury or continuous hemorrhaging was excluded. Then $50 \mu \mathrm{l}$ of $2 \%$ hyaluronic acid hydrogel (5-mm-diameter cylinder) with $1 \times 10^{5}$ human ATSCs or BMSCs was immediately implanted into the defect cavity. The periosteum and scalp were closed by suture. Animals were allowed to move following recovery from the anesthesia and were sacrificed by overdose of pentobarbital 6 weeks after surgery. The defect sites were removed, including sufficient parietal bone and soft connective tissues surrounding the defect areas.

\section{Micro-computed tomography imaging analysis}

Micro-computed tomography (microCT) was used for quantitative evaluation of the bone formation. The samples were imaged using a high-resolution $70-\mathrm{kVp}$ scan by microCT machine (VivaCT; Scanco Medical, Bassersdorf, Switzerland). The 3D reconstruction was performed using standardized segmentation parameters (sigma 0.8, threshold 160-1000), which were kept constant through the scan. Circular contour lines were drawn around the defect area (diameter $=5 \mathrm{~mm}$ ) excluding the neighboring native bone. The 3D reconstructive images of samples were generated from 2D slices by machine built-in software. The bone volume within the selected circular defect was calculated using the quantitative $3 \mathrm{D}$ evaluation program included in the microCT software package.

\section{Histology and immunohistochemistry}

Immunohistochemical staining was performed as described previously [23]. The samples were washed in PBS, fixed in $4 \%$ paraformaldehyde, decalcified, dehydrated, and embedded in paraffin. Sections were cut at a thickness of $5 \mu \mathrm{m}$ and were stained with HE after deparaffination. Endogenous peroxidase activity was quenched with $3 \%$ hydrogen peroxide for $20 \mathrm{~min}$ at room temperature. Antigen retrieval was then performed with citrate buffer at $80^{\circ} \mathrm{C}$ for 10 min for immunohistochemistry detection. Primary antibody against osteocalcin (1:100, sc-365797; Santa Cruz, CA, USA) was used. Donkey anti-goat IgG horseradish peroxidase (HRP)-conjugated secondary antibody was then added for 1 hour, followed by $3,3^{\prime}$-diaminobenzidine tetrahydrochloride (DAKO, Glostrup, Denmark) in the presence of $\mathrm{H}_{2} \mathrm{O}_{2}$ for signal detection of osteocalcin. Afterward, the sections were rinsed, counterstained in hematoxylin, dehydrated with graded ethanol and xylene, and mounted with $p$-xylene-bis-pyridinium bromide (DPX) permount (Sigma-Aldrich). Primary antibody was replaced with blocking solution in the negative controls. All incubation times and conditions were strictly controlled. The sections were examined under light microscopy (DMRXA2; Leica Microsystems Wetzlar GmbH, Germany).

\section{Data analysis}

All experiments were performed at least three times. All data were expressed as the mean \pm SD. The data were analyzed by independent two-tailed Student's $t$ test using SPSS (version16.0; Chicago, IL, USA). $p<0.05$ was regarded as statistically significant.

\section{Results}

Characterize ATSCs and BMSCs with flow cytometry

The surface antigens of human ATSCs and BMSCs were detected by flow cytometry. The results showed that the 


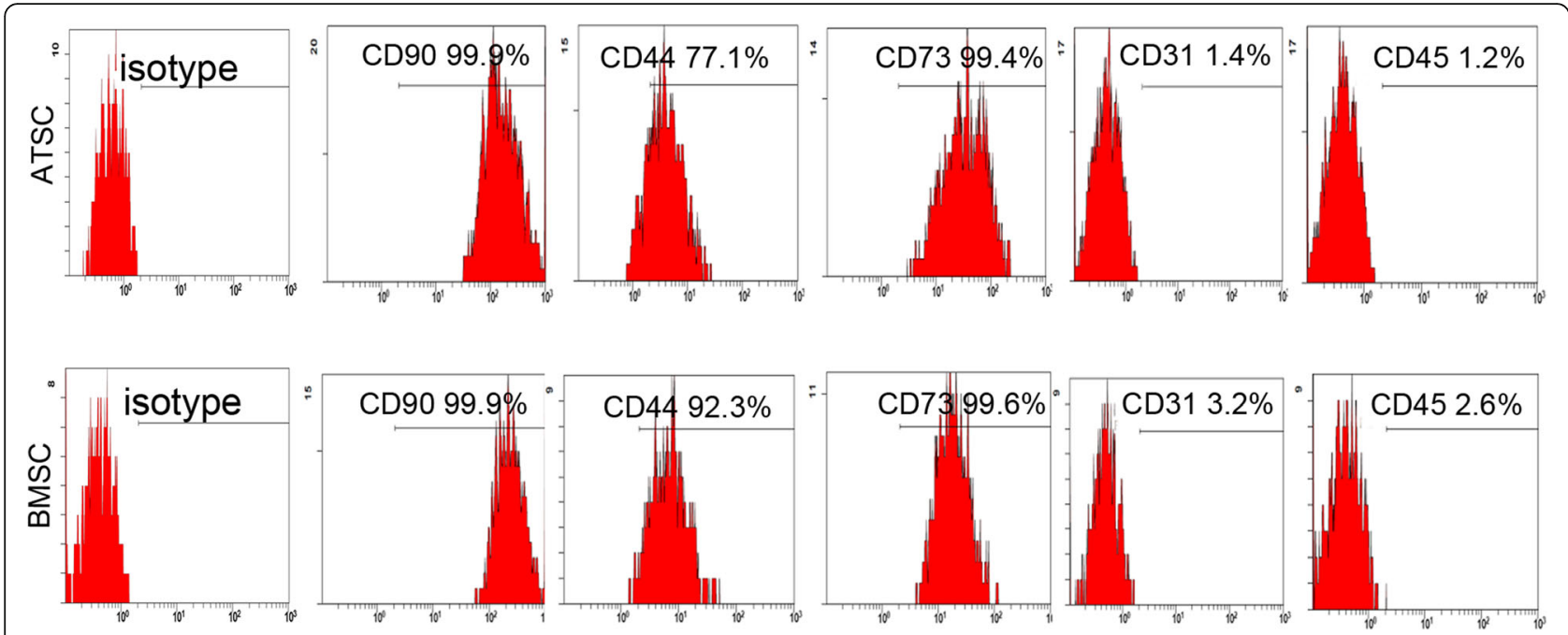

Fig. 1 Characterization of cell surface markers of ATSCs and BMSCs. Cell surface markers of ATSCs and BMSCs (both at passage 3) were analyzed using flow cytometry. Antibodies against CD90, CD44, CD73, CD31, and CD45 were used to characterize ATSCs and BMSCs. ATSC: adipose tissuederived MSC, BMSC: bone marrow-derived MSC, MSC: mesenchymal stem cell
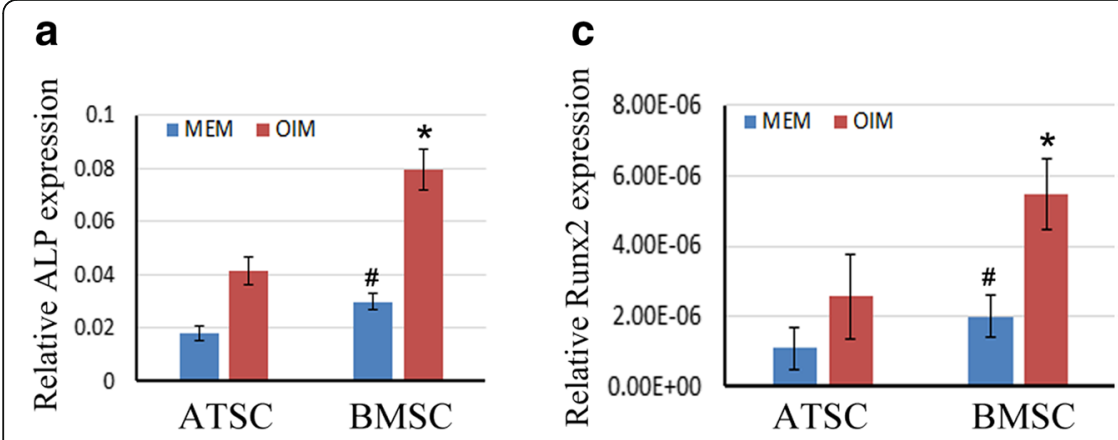

e
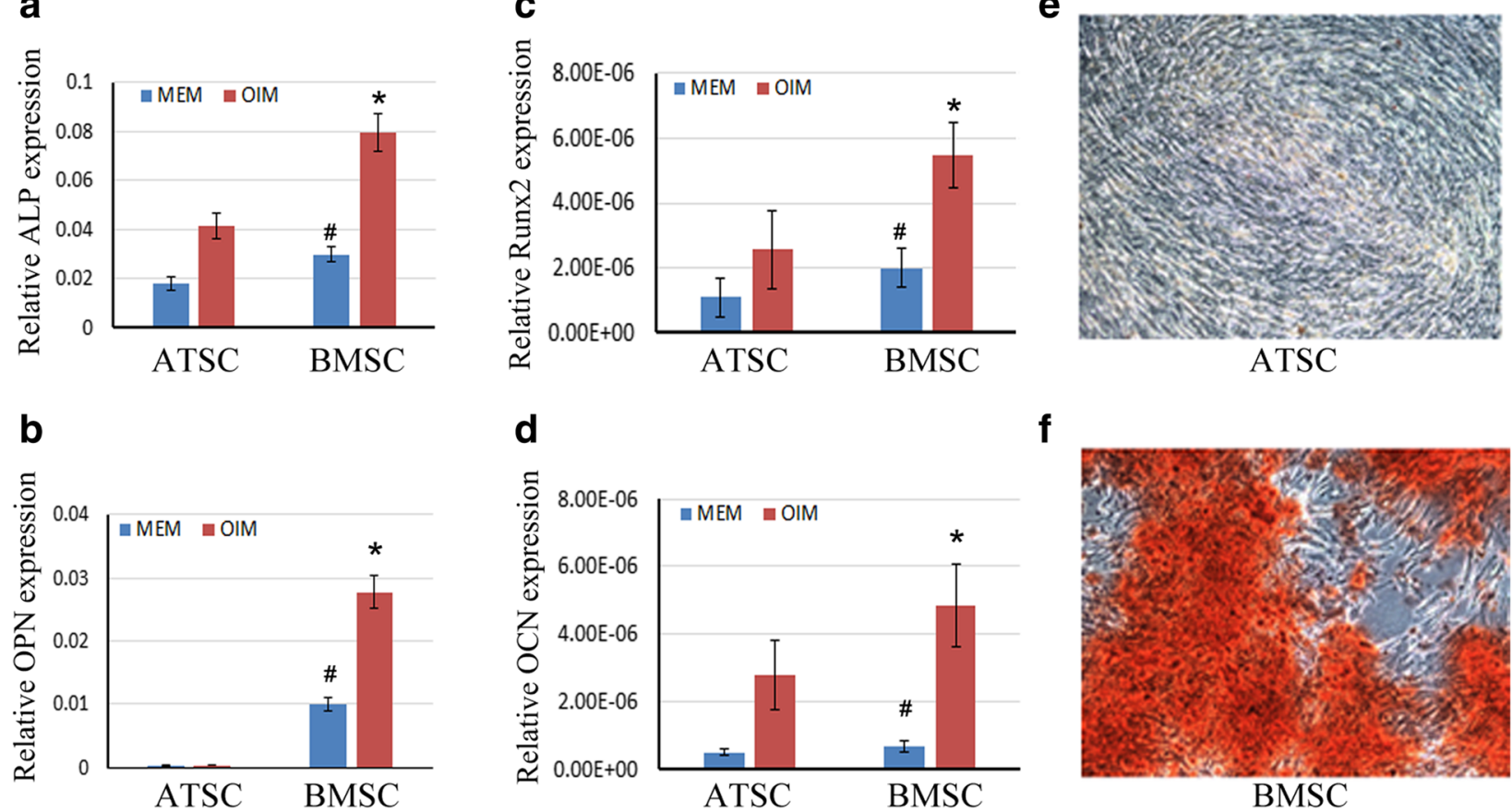

$\mathbf{f}$

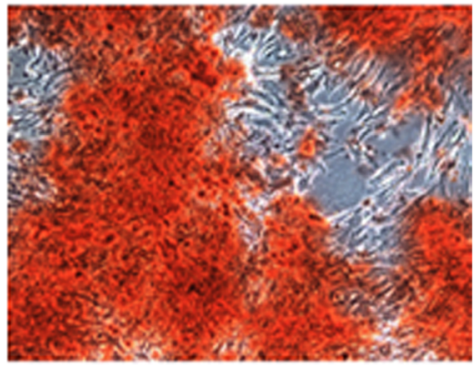

BMSC

Fig. 2 Difference in osteogenesis ability between ATSCs and BMSCs in vitro. a-d Total RNA extracted from ATSCs and BMSCs or cells subjected to OIM for 7 days. Relative expression levels of ALP, Runx2, OPN, and OCN checked by qRT-PCR. $\beta$-actin as an internal control. Data expressed as mean \pm SD $(n=3) .{ }^{*} p<0.05$, compared with MSCs in OIM; \#p < 0.05, compared with MSCs in a-MEM. e, $\mathbf{f}$ Alizarin Red S staining of calcium deposits formed by ATSCs and BMSCS. ATSCs and BMSCs cultured in OIM for 14 days, then cells fixed and stained with Alizarin Red S. ALP: alkaline phosphatase, ATSC: adipose tissue-derived MSC, BMSC: bone marrow-derived MSC, MEM: minimum essential medium, MSC: mesenchymal stem cell, OCN: Osteocalcin, OIM: osteogenic induction medium, OPN: Osteopontin, Runx2: runt-related transcription factor 2 
cells were positive for CD90, CD44, and CD73 and negative for CD31 and CD45 (Fig. 1). The data showed that the cells expressed typical surface markers of MSCs and therefore were used for the experiments described in the following.

\section{Compare osteogenesis of ATSCs and BMSCs in vitro}

In order to compare the osteogenic differentiation potential capacities of ATSCs and BMSCs, MSCs were treated with OIM for several days and then the mRNA expression levels of genes related to osteogenesis were detected by quantitative real-time RT-PCR (qRT-PCR). As shown in Fig. 2a-d, the expression levels of alkaline phosphatase (ALP) and runt-related transcription factor 2 (Runx2), which are early markers for osteogenic commitment, were markedly increased in BMSCs compared with the ATSCs, as well as the late osteogenic markers Osteocalcin (OCN) and Osteopontin (OPN). To confirm the osteogenic commitment of BMSCs and ATSCs, Alizarin Red S staining was used to detect the formation of calcium deposit. The results showed that after 14 days of OIM induction, mineralization was seen in BMSCs upon osteogenic induction, while there were very few Alizarin Red S-positive calcium nodules formed in the ATSC group (Fig. 2e, f). These data indicated that BMSCs possessed higher potential for differentiation into osteoblasts compared to ATSCs.

\section{Compare adipogenesis of ATSCs and BMSCs in vitro}

Next, we evaluated the adipogenic differentiation potential capacities of ATSCs and BMSCs. The cells were treated with AIM for several days, and then the mRNA expression levels of genes related to adipogenesis were detected by qRT-PCR. Our results showed that the expression levels of adipogenesis-related marker genes such as peroxisome proliferatoractivated receptor gamma (PPAR $\gamma)$, CCAAT/enhancer-binding protein alpha $(\mathrm{CEBP \alpha})$, adipocyte protein 2 (AP2), and lipoprotein lipase (LPL) were significantly increased in ATSCs compared with the BMSCs (Fig. 3a-d). After 21 days of AIM induction, the cells were fixed for Oil Red $\mathrm{O}$ staining. The result showed that BMSCs had lower adipogenic differentiation potential as compared with ATSCs (Fig. 3e, f).

\section{DNA methylation analysis of main transcription factors}

Because epigenetic regulation is an important factor to control MSC differentiation and the methylation status of DNA is the most common epigenetic modification of the genome in mammalian cells [24], we asked whether DNA

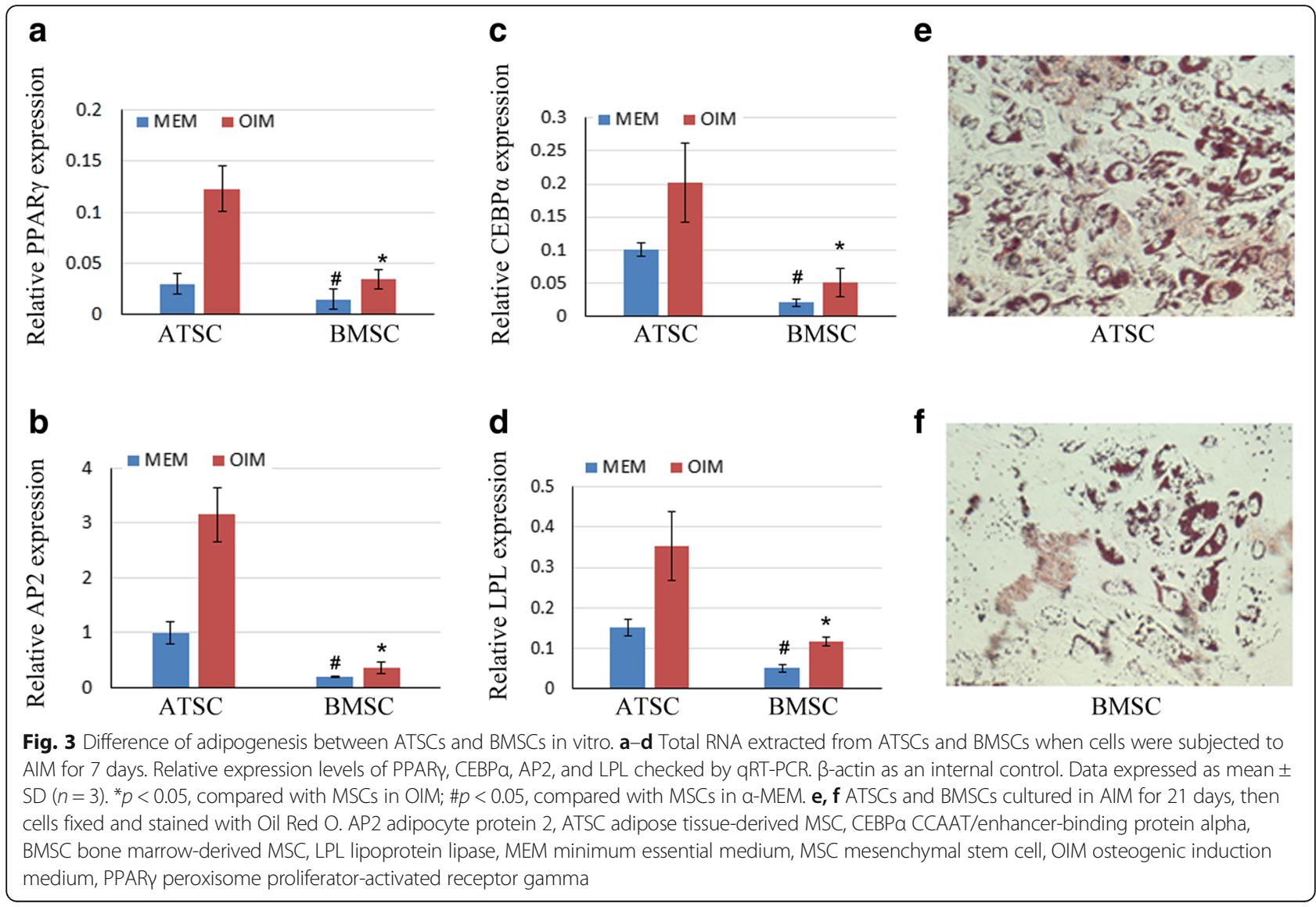


methylation was involved in fate determination of ATSCs and BMSCs. Runx2 and PPARY are the main master transcriptional factors controlling osteogenesis and adipogenesis, respectively. So, revealing the DNA methylation status of these two transcription factors may demonstrate their relationship with MSC fate determination. We calculated the percentage of methylated $\mathrm{CpG}$ loci (percent $\mathrm{CpG}$ methylation) in the total four CpG loci in Runx2 promoter and in four CpG loci in PPARy promoter, respectively. We found that Runx 2 promoter was hypermethylated whereas PPAR $\gamma$ promoter was hypomethylated in ATSCs (75\% and 25\% CpG methylation) (Fig. 4a, b). On the other hand, the methylation status of Runx2 was hypomethylated and PPARY promoter was hypermethylated. These data suggest that DNA demethylation could be involved, at least partially, in the regulation of Runx2 and PPARy in ATSCs and BMSCs; the source of MSCs is a direct factor influencing fate determination of MSCs.

\section{Compare chondrogenesis of ATSCs and BMSCs in vitro}

Next, we wanted to know whether there is any difference in chondrogenesis ability between ATSCs and BMSCs. a
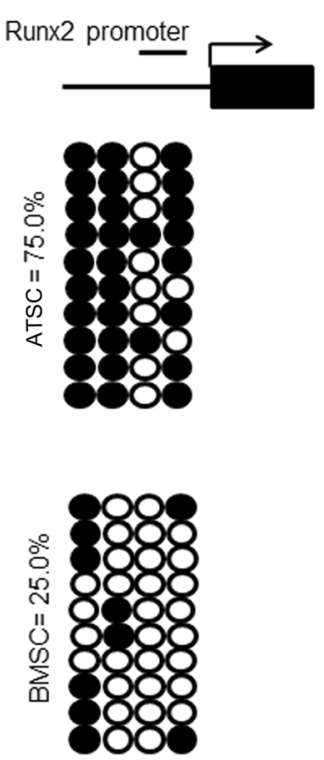

Methylated CpG b
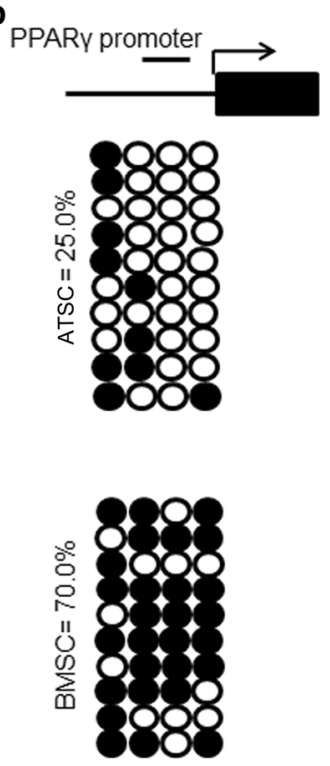

O Unmethylated $\mathrm{CpG}$
Fig. 4 DNA methylation status of Runx2 and PPARy in ATSCs and BMSCs. DNA methylation status of Runx2 (a) and PPARY (b) promoters in ATSCs and BMSCs were examed using sodium bisulfite sequencing. Top panel indicates CpG dinucleotide position of the Runx2 and PPARY promoter regions. Each PCR product was subcloned and subjected to nucleotide sequencing analysis. Ten representative sequenced clones depicted by filled (methylated) and open (unmethylated) circles for each CPG site. ATSC: adipose tissue-derived MSC, BMSC: bone marrow-derived MSC, MSC: mesenchymal stem cell, PPARY: peroxisome proliferator-activated receptor gamma, Runx2: runt-related transcription factor 2
The cells were treated with CIM for 10 days, and then the expression levels of Sox 9 and Collagen type II were evaluated by qRT-PCR. The results showed that both Sox9 and Collagen type II were slightly lower in ATSCs (Fig. 5a, b). Further bisulfite sequencing data showed that there was no significant difference in the methylation status of CpG sites in the promoter of Sox 9 between ATSCs and BMSCs (Fig. 5c).

\section{Ectopic bone formation of ATSCs and BMSCs in vivo}

To further evaluate the advantages of BMSCs in osteogenic differentiation in vivo, BMSCs and ATSCs were loaded onto sterilized Skelite ${ }^{ø}$ resorbable Si-TCP bone graft substitutes respectively and implanted subcutaneously at the dorsal sides of nude mice. The transplants were harvested 8 weeks later and subjected to histological examination with HE staining or immunohistochemical analysis to detect the distribution of osteoid and the expression of OCN. Our results showed that transplantation of BMSCs with $\mathrm{Si}-\mathrm{TCP}$ resulted in more bone-like tissue formation and less loose fibrous tissue and adipose tissue formation around the scaffold compared to the ATSCs with Si-TCP in nude mice. The formation of bone-like tissue was confirmed by the presence of osteocalcin (Fig. 6a, b). These results indicated that BMSCs were superior to ATSCs in ectopic bone formation in vivo.

\section{Bone regeneration in vivo using the calvarial defect model}

To compare the effect of ATSCs and BMSCs on bone repair, the nude mice calvarial bone critical defect model was used. Then $50 \mu \mathrm{l}$ of $2 \%$ hyaluronic acid hydrogel (5-mm-diameter cylinder) with $1 \times 10^{5} \mathrm{hu}$ man ATSCs or BMSCs was immediately implanted into the defect cavity. Six weeks later, the samples were collected for X-ray and microCT reconstruction analysis. The result showed that more new bone tissue was observed in the BMSC group compared with the ATSC group (Fig. 7a, b). The ratio of bone volume/total volume in the BMSC group was significantly increased compared to that of the ATSC group (Fig. 7a, b).

\section{Discussion}

In the present study, we compared the differentiation capacities of ATSCs and BMSCs, and demonstrated that BMSCs possessed stronger osteogenic but lower adipogenic differentiation potentials compared to ATSCs. There was no significant difference between their chondrogenic differentiation potential. Interestingly, our results provided important evidence that the DNA methylation status of the master transcription factors controlling MSC fate determination was responsible for 
a

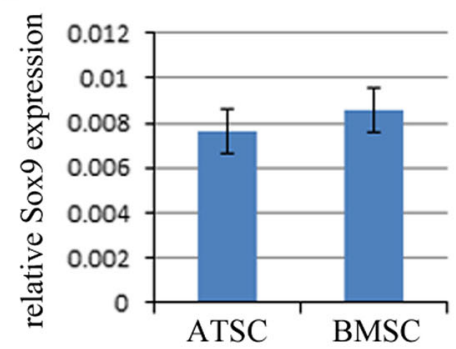

b

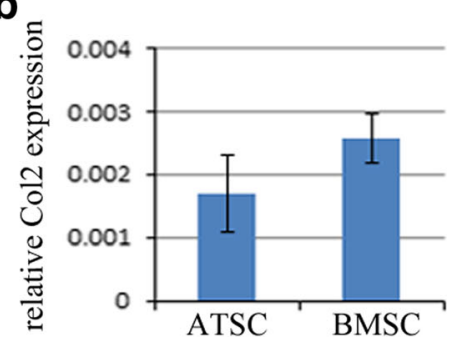

c
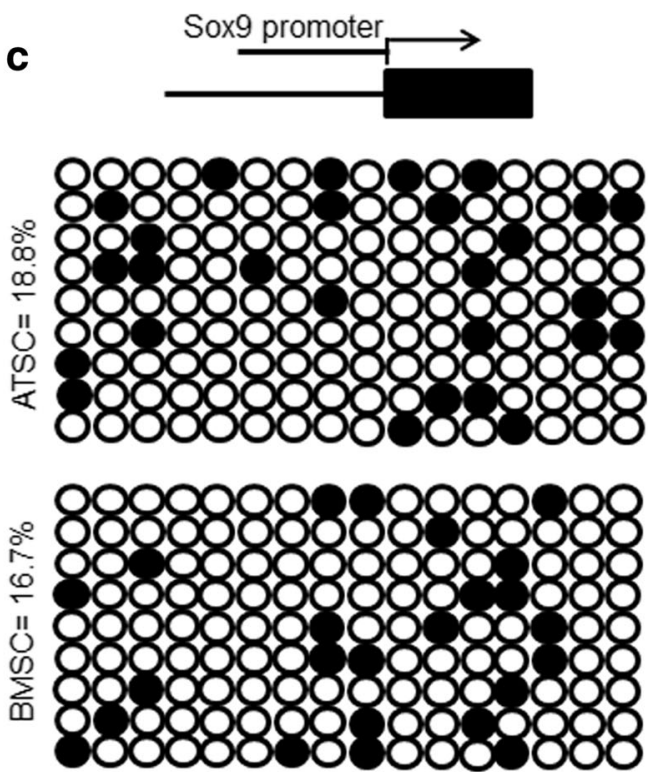

Fig. 5 BMSCs showed slightly stronger chondrogenic differentiation potential. $\mathbf{a}$, b Total RNA extracted from ATSCs and BMSCs when cells were subjected to CIM for 10 days. Relative expression levels of Sox 9 and Col 2 checked by qRT-PCR. $\beta$-actin as an internal control. Data expressed as mean \pm SD. $p<0.05$. c DNA methylation status of Sox9 promoter in ATSCs and BMSCs using sodium bisulfite sequencing. Top panel indicates CpG dinucleotide position of the Sox9 promoter region and numbers show positions of CpGs relative to the translation start site. Each PCR product was subcloned and subjected to nucleotide sequencing analysis. Ten representative sequenced clones depicted by filled (methylated) and open (unmethylated) circles for each CpG site. ATSC: adipose tissue-derived MSC, Col2: Collagen type II, BMSC: bone marrow-derived MSC, MSC: mesenchymal stem cell

the regulation of MSC differentiation capacities. The epigenetic memory obtained from either bone marrow or adipose tissue favored their differentiation along osteoblastic or adipocytic lineage. This is of particular interest, since the finding provided a potential explanation to elucidate the mechanism which was responsible for the regulation of MSC differentiation capacities by the origin they derived.

MSCs have been isolated from the umbilical cord, umbilical cord blood, bone marrow, adipose tissue, and many other adult tissues. They have been reported to share similar characteristics in vitro, such as plastic adherence, proliferation capacity, immunophenotype, and multilineage differentiation ability [25]. Lee et al. [26] reported that ATSCs were superior to BMSCs with respect to their maintenance of proliferating ability, but had similar morphology, phenotype, and microarray analysis of gene expression, and did not reveal differentially expressed osteogenic or adipogenic related genes between ATSCs and BMSCs. Our results have also provided evidence that ATSCs and BMSCs have similar morphology and cell surface markers, but their differentiation capacity was different. However, the intrinsic molecular mechanism supporting these observations has been lacking. We have partially addressed this question by exploring the methylation status of promoters of main transcription factors regulating trilineage differentiation potentials of MSCs.
DNA methylation is one of the most important epigenetic regulations which possesses the power to control the differentiation of or maintain the self-renewal of MSCs [17]. Accumulating evidence has demonstrated that changes in the methylation states of the CpG islands in the promoter regions or the first exon are inversely responsible for expression of the corresponding genes [27, 28]. The bivalent loci in MSCs are often reduced in DNA methylation and can be further methylated or activated, distinct from those in embryonic stem cells and differentiated cells [18]. A recent study demonstrated that the promoter regions of key genes in osteogenic differentiation such as BMP2 and ALP are epigenetically locked in MSCs to prevent their expression in nonosteogenic cells [29].

Sørensen et al. [30] reported that MSC differentiation did not affect lineage-specific promoter methylation states, arguing that these methylation patterns in differentiated cells are already established at the progenitor stage. But they did not compare the methylation status of the main transcription factors from different sourcederived MSCs. We produced bisulfite PCR analysis to check the DNA methylation status of Runx2, PPAR $\gamma$, and Sox9 from ATSCs and BMSCs. We demonstrated that BMSCs possessed stronger osteogenic and lower adipogenic differentiation potentials, which is completely different from ATSCs. The conclusion is that the 


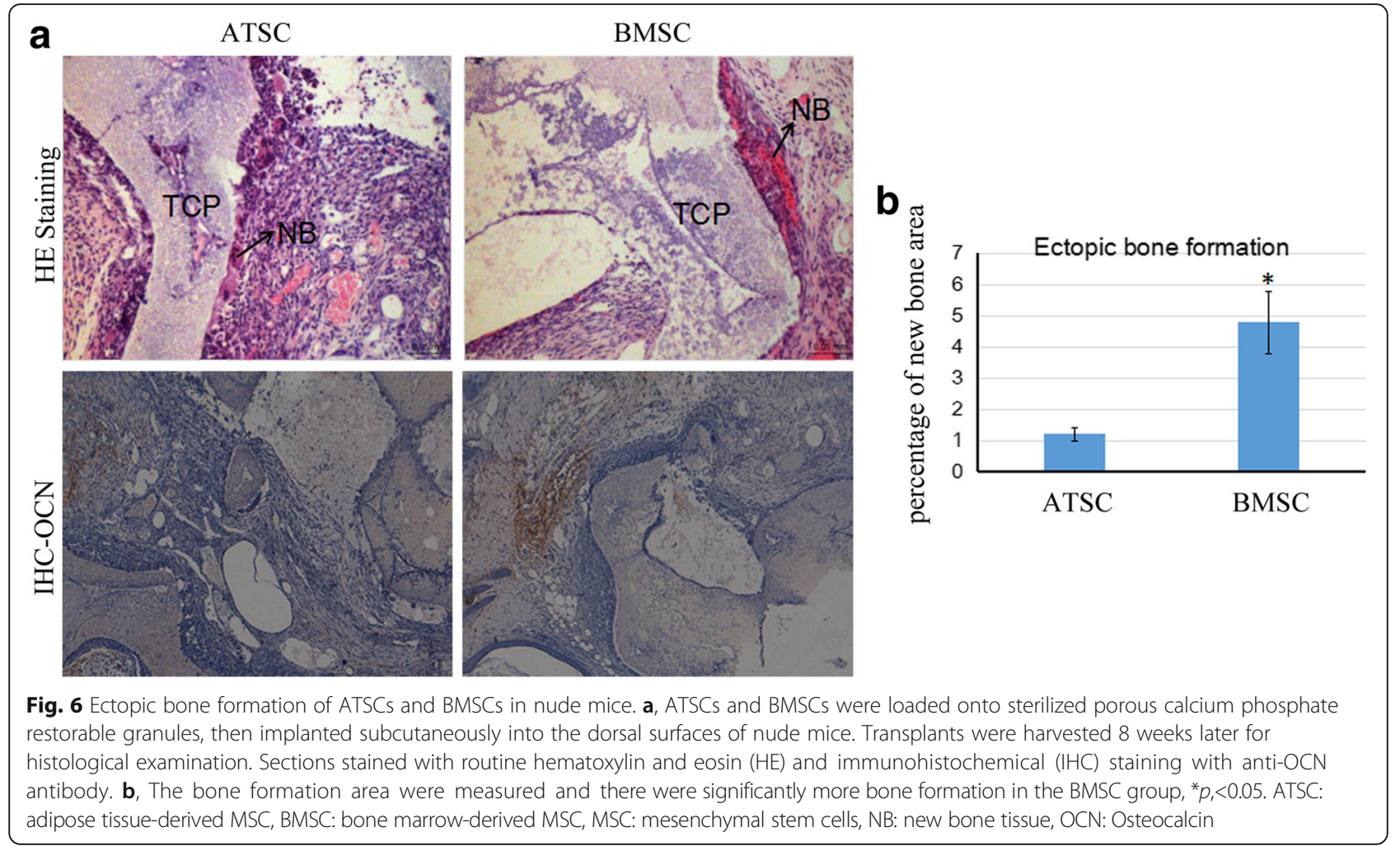

differentiation potential of MSCs is highly influenced by their tissue of origin through epigenetic regulations such as DNA methylation of important transcription factors.

MSCs hold great promise for the treatment of a variety of difficult diseases such as myocardial infarction [31], neural diseases [32], and bone and cartilage defect [33, 34]. Bone marrow is the one of the major sources of MSCs, where they represent only approximately $0.001-0.01 \%$ of the nucleated cells, which is much less abundant than hematopoietic stem cells (HSCs). Adipose tissue is the main alternative source of MSCs and is more abundant and easier to isolate. Which type of MSCs is more suitable for clinical application? Some studies indicate that the clinical application potential of ATSCs is more effective or as effective as

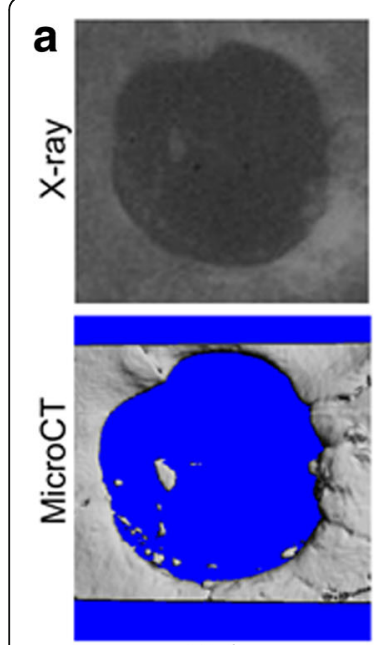

Gel
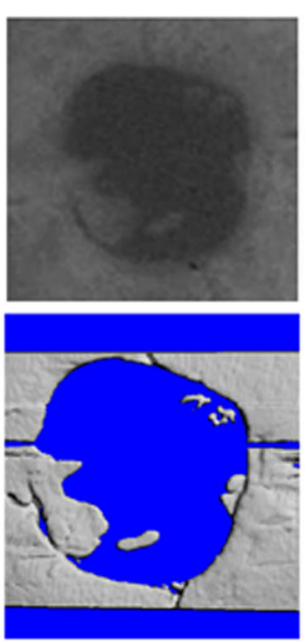

Gel+ATSC
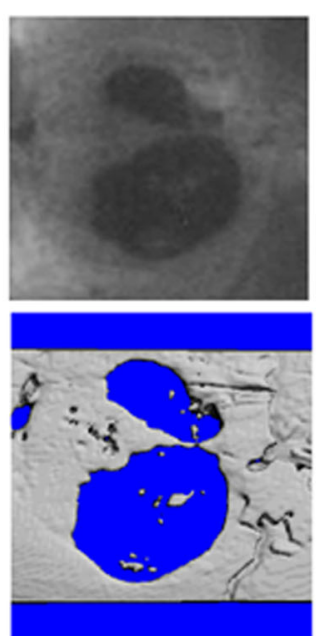

Gel+BMSC

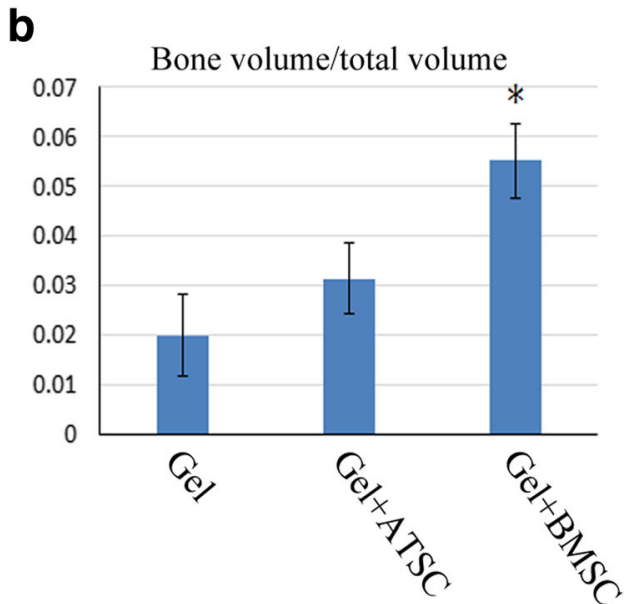

Fig. 7 ATSCs and BMSCs enhanced calvarial bone repair in nude mice. a X-ray and microCT analysis 3D reconstruction of calvarial bone samples. b MicroCT analysis showed the new bone volume in the BMSC group was significantly increased (*) compared to that of the control group. ATSC adipose tissue-derived MSC, BMSC bone marrow-derived MSC, CT computed tomography, MSC mesenchymal stem cell 
that of BMSCs, while others conclude that BMSCs are superior to ATSCs [11-13]. In this study, we isolated bone marrow and adipose-derived MSCs from people aged older than 60 years. ATSCs and BMSCs from the same donors were evaluated. The results showed that BMSCs and ATSCs exhibited different trilineage differentiation potentials, although they expressed similar cell surface makers and had a similar phenotype. The bisulfite sequencing data further provided a mechanism basis to make this conclusion, which explicated why MSCs from bone marrow were better for bone regeneration. The differential ability in trilineage differentiation is determined by the origin of MSCs. Checking DNA methylation of the main transcription factors governing MSC differentiation may be a predictive approach to distinguish different subpopulations of MSCs which may lead to better outcome for tissue regeneration. For example, MSCs with a lower DNA methylation rate in Runx2 promoter and a higher methylation rate in PPAR $\gamma$ promoter would result in better bone regeneration.

\section{Conclusion}

Taken together, our results demonstrated that the methylation status of the main transcription factors controlling MSC fate influenced their expression, which resulted in the different differentiation capacities of ATSCs and BMSCs. This study has provided strong evidence that BMSCs are superior to ATSCs in terms of osteogenic differentiation rather than adipogenic differentiation, which is meaningful for the application of BMSCs in tissue engineering and regeneration of bone instead of ATSCs.

\section{Additional file}

Additional file 1: Table S1. Sequences of primers for real-time PCR analysis, Table S2. Sequences of primers for bisulfite sequencing analysis, and Table S3. Sequences of primers for CHIP-PCR analysis.

\section{Abbreviations}

AIM: Adipogenic induction medium; ALP: Alkaline phosphatase; AP2: Adipocyte protein 2; ATSC: Adipose tissue-derived MSC; BMSC: Bone marrow-derived MSC; CEBPa: CCAAT/enhancer-binding protein alpha; CIM: Chondrogenic induction medium; HSC: Hematopoietic stem cell; LPL: Lipoprotein lipase; MSC: Mesenchymal stem cell; OCN: Osteocalcin; OIM: Osteogenic induction medium; OPN: Osteopontin; PBS: Phosphate-buffered saline; PPARY: Peroxisome proliferator-activated receptor gamma; Runx2: Runt-related transcription factor 2; a-MEM: Minimum Essential Media alpha

\section{Funding}

The work was partially supported by grants from Hong Kong Government Research Grant Council, General Research Fund (14119115, 14160917, 9054014 N_CityU102/15 and T12-402/17-N); National Natural Science Foundation of China (81371946, 81430049 and 81772322); Hong Kong Innovation Technology Commission Funds (ITS/UIM-305); Shenzhen City Science and Technology Bureau, China (JCYJ20150630165236960). This study was also supported in part by SMART program, Lui Che Woo Institute of Innovative Medicine, The Chinese University of Hong Kong and the research was made possible by resources donated by Lui Che Woo Foundation Limited.
Availability of data and materials

All of the supporting data can be downloaded.

\section{Authors' contributions}

BW (Guangzhou University of Traditional Chinese Medicine) and GL conceived and designed the study. LLX and YML carried out the experimentation and wrote the first draft of the manuscript. YXS and BW (Chinese University of Hong Kong) performed the animal experiments. YPX and WPL collected clinical samples and cultured BMSCS and ATSCS. QSW participated in data interpretation. HBW and WH participated in the design of the study and assisted in data interpretation. All authors read and approved the final manuscript.

\section{Authors' information}

Not applicable.

\section{Ethics approval and consent to participate}

Human ethics approval was obtained from the Joint CUHK-NTEC Clinical Research Ethics Committee of the Chinese University of Hong Kong (Reference No: CRE2011.383). Animal surgery was carried out under the animal license issued by the Hong Kong SAR Government and the approval of the Animal Experimentation Ethics Committee of the Chinese University of Hong Kong (Ref No: 16-109-GRF). Human adipose tissue and bone marrow samples of three patients (females 60-65 years old) with total hip arthroplasty who gave informed consent were obtained from The Third Affiliated Hospital of Guangzhou University of Chinese Medicine (Guangzhou, China).

\section{Consent for publication}

All the authors give their consents for publication in the journal.

\section{Competing interests}

The authors declare that they have no competing interests.

\section{Publisher's note}

Springer Nature remains neutral with regard to jurisdictional claims in published maps and institutional affiliations.

\section{Author details}

${ }^{1}$ Key Laboratory of Orthopaedics \& Traumatology, The First Affiliated Hospital of Guangzhou University of Chinese Medicine, The First Clinical Medical College, Guangzhou University of Chinese Medicine, Guangzhou, China. 2Departments of Diagnostics of Traditional Chinese Medicine, Guangzhou University of Traditional Chinese Medicine, Guangzhou, Guangdong 510006, People's Republic of China. ${ }^{3}$ Department of Orthopaedics \& Traumatology, Faculty of Medicine, The Chinese University of Hong Kong, Prince of Wales Hospital, Shatin, Hong Kong, Special Administrative Region of China. ${ }^{4}$ Key Laboratory for Regenerative Medicine, Ministry of Education, School of Biomedical Sciences, Faculty of Medicine, The Chinese University of Hong Kong, Hong Kong, SAR, China. ${ }^{5}$ Stem Cells and Regenerative Medicine Laboratory, Lui Che Woo Institute of Innovative Medicine, Li Ka Shing Institute of Health Sciences, The Chinese University of Hong Kong, Prince of Wales Hospital, Shatin, Hong Kong, Special Administrative Region of China. ${ }^{6}$ The CUHK-ACC Space Medicine Centre on Health Maintenance of Musculoskeletal System, The Chinese University of Hong Kong Shenzhen Research Institute, Shenzhen, People's Republic of China. 'Department of Traumatology, The Third Affiliated Hospital of Guangzhou University of Traditional Chinese Medicine, Guangzhou, Guangdong 510240, People's Republic of China. ${ }^{8}$ Room 904, 9/F, Li Ka Shing Institute of Health Institute, Prince of Wales Hospital, The Chinese University of Hong Kong, Shatin, Hong Kong, Special Administrative Region of China. ${ }^{9}$ Department of Traumatology, The First Affiliated Hospital of Guangzhou University of Traditional Chinese Medicine, Guangzhou, China.

Received: 20 February 2017 Revised: 9 August 2017 Accepted: 30 October 2017 Published online: 06 December 2017

\section{References}

1. Parekkadan B, Milwid JM. Mesenchymal stem cells as therapeutics. Annu Rev Biomed Eng. 2010;12:87-117.

2. Hicks AU, Lappalainen RS, Narkilahti S, Suuronen R, Corbett D, Sivenius J, et al. Transplantation of human embryonic stem cell-derived neural precursor cells 
and enriched environment after cortical stroke in rats: cell survival and functional recovery. Eur J Neurosci. 2009;29(3):562-74.

3. Li Y, Chen J, Chen XG, Wang L, Gautam SC, Xu YX, et al. Human marrow stromal cell therapy for stroke in rat: neurotrophins and functional recovery. Neurology. 2002;59(4):514-23.

4. Swanger SA, Neuhuber B, Himes BT, Bakshi A, Fischer I. Analysis of allogeneic and syngeneic bone marrow stromal cell graft survival in the spinal cord. Cell Transplant. 2005;14(10):775-86.

5. Chen J, Sanberg PR, Li Y, Wang L, Lu M, Willing AE, et al. Intravenous administration of human umbilical cord blood reduces behavioral deficits after stroke in rats. Stroke. 2001;32(11):2682-8.

6. Hoogduijn MJ, Dor FJMF. Mesenchymal stem cells: are we ready for clinical application in transplantation and tissue regeneration? Front Immunol. 2013:4:144.

7. Puissant B, Barreau C, Bourin P, Clavel C, Corre J, Bousquet C, et al. Immunomodulatory effect of human adipose tissue-derived adult stem cells: comparison with bone marrow mesenchymal stem cells. $\mathrm{Br}$ J Haematol. 2005;129(1):118-29.

8. Chung CS, Fujita N, Kawahara N, Yui S, Nam E, Nishimura R. A comparison of neurosphere differentiation potential of canine bone marrow-derived mesenchymal stem cells and adipose-derived mesenchymal stem cells. J Vet Med Sci. 2013;75(7):879-86.

9. Cooper GM, Durham EL, Cray Jr JJ, Bykowski MR, DeCesare GE, Smalley MA, et al. Direct comparison of progenitor cells derived from adipose, muscle, and bone marrow from wild-type or craniosynostotic rabbits. Plast Reconstr Surg. 2011;127(1):88-97.

10. Danisovic L, Varga I, Polak S, Ulicna M, Hlavackova L, Bohmer D, et al. Comparison of in vitro chondrogenic potential of human mesenchymal stem cells derived from bone marrow and adipose tissue. Gen Physiol Biophys. 2009;28(1):56-62.

11. Elman JS, Li M, Wang F, Gimble JM, Parekkadan B. A comparison of adipose and bone marrow-derived mesenchymal stromal cell secreted factors in the treatment of systemic inflammation. J Inflamm (Lond). 2014;11(1):1.

12. Rasmussen JG, Frobert O, Holst-Hansen C, Kastrup J, Baandrup U, Zachar V, et al. Comparison of human adipose-derived stem cells and bone marrow-derived stem cells in a myocardial infarction model. Cell Transplant. 2014;23(2):195-206.

13. Huang Jl, Kazmi N, Durbhakula MM, Hering TM, Yoo JU, Johnstone B. Chondrogenic potential of progenitor cells derived from human bone marrow and adipose tissue: a patient-matched comparison. J Orthop Res. 2005;23(6):1383-9.

14. Hsiao ST, Asgari A, Lokmic Z, Sinclair R, Dusting GJ, Lim SY, et al. Comparative analysis of paracrine factor expression in human adult mesenchymal stem cells derived from bone marrow, adipose, and dermal tissue. Stem Cells Dev. 2012;21(12):2189-203.

15. Ahmadian Kia N, Bahrami AR, Ebrahimi M, Matin MM, Neshati Z, Almohaddesin MR, et al. Comparative analysis of chemokine receptor's expression in mesenchymal stem cells derived from human bone marrow and adipose tissue. J Mol Neurosci. 2011;44(3):178-85.

16. Rui $Y, X u L$, Chen $R$, Zhang $T$, Lin $S$, Hou Y, et al. Epigenetic memory gained by priming with osteogenic induction medium improves osteogenesis and other properties of mesenchymal stem cells. Sci Rep. 2015:5:11056.

17. Boquest AC, Noer A, Collas P. Epigenetic programming of mesenchymal stem cells from human adipose tissue. Stem Cell Rev. 2006:2(4):319-29.

18. Leu YW, Huang TH, Hsiao SH. Epigenetic reprogramming of mesenchymal stem cells. Adv Exp Med Biol. 2013;754:195-211.

19. Hsiao SH, Lee KD, Hsu CC, Tseng MJ, Jin VX, Sun WS, et al. DNA methylation of the Trip10 promoter accelerates mesenchymal stem cell lineage determination. Biochem Biophys Res Commun. 2010;400(3):305-12.

20. Zuk PA, Zhu M, Mizuno H, Huang J, Futrell JW, Katz AJ, et al. Multilineage cells from human adipose tissue: implications for cell-based therapies. Tissue Eng. 2001;7(2):211-28.

21. Haynesworth SE, Baber MA, Caplan Al. Cell surface antigens on human marrow-derived mesenchymal cells are detected by monoclonal antibodies. Bone. 1992;13(1):69-80.

22. Zinn RL, Pruitt K, Eguchi S, Baylin SB, Herman JG. hTERT is expressed in cancer cell lines despite promoter DNA methylation by preservation of unmethylated DNA and active chromatin around the transcription start site. Cancer Res. 2007:67(1):194-201.

23. Rui YF, Lui PP, Lee YW, Chan KM. Higher BMP receptor expression and BMP2-induced osteogenic differentiation in tendon-derived stem cells compared with bone-marrow-derived mesenchymal stem cells. Int Orthop. 2012:36(5):1099-107.

24. Poloni A, Maurizi G, Leoni P, Serrani F, Mancini S, Frontini A, et al. Human dedifferentiated adipocytes show similar properties to bone marrowderived mesenchymal stem cells. Stem Cells. 2012;30(5):965-74.

25. Meirelles LD, Caplan Al, Nardi NB. In search of the in vivo identity of mesenchymal stem cells. Stem Cells. 2008;26(9):2287-99.

26. Lee RH, Kim B, Choi I, Kim H, Choi HS, Suh K, et al. Characterization and expression analysis of mesenchymal stem cells from human bone marrow and adipose tissue. Cell Physiol Biochem. 2004;14(4-6):311-24.

27. Wagner JR, Busche S, Ge B, Kwan T, Pastinen T and Blanchette M. The relationship between DNA methylation, genetic and expression interindividual variation in untransformed human fibroblasts. Genome Biology. 2014;15(2):R37.

28. Schultz MD, He YP, Whitaker JW, Hariharan M, Mukamel EA, Leung D, et al. Human body epigenome maps reveal noncanonical DNA methylation variation. Nature. 2015;523(7559):212-6.

29. Cho YD, Yoon WJ, Kim WJ, Woo KM, Baek JH, Lee G, et al. Epigenetic modifications and Canonical Wingless/int-1 Class (WNT) signaling enable trans-differentiation of nonosteogenic cells into osteoblasts. J Biol Chem. 2014;289(29):20120-8.

30. Sorensen AL, Timoskainen S, West FD, Vekterud K, Boquest AC, AhrlundRichter $L$, et al. Lineage-specific promoter DNA methylation patterns segregate adult progenitor cell types. Stem Cells Dev. 2010;19(8):1257-66.

31. Joggerst SJ, Hatzopoulos AK. Stem cell therapy for cardiac repair: benefits and barriers. Expert Rev Mol Med. 2009:11:e20

32. Martino G, Franklin RJM, Baron-Van Evercooren A, Kerr DA, STEM SCMS. Stem cell transplantation in multiple sclerosis: current status and future prospects. Nat Rev Neurol. 2010;6(5):247-55.

33. Huang $S$, Xu LL, Zhang YF, Sun YX, Li G. Systemic and local administration of allogeneic bone marrow-derived mesenchymal stem cells promotes fracture healing in rats. Cell Transplant. 2015;24(12):2643-55.

34. Lin WP, Xu LL, Zwingenberger S, Gibon E, Goodman S, Gang L. Mesenchymal stem cells homing to improve bone healing. J Orthop Translat. 2017:9:19-27.

\section{Submit your next manuscript to BioMed Central and we will help you at every step:}

- We accept pre-submission inquiries

- Our selector tool helps you to find the most relevant journal

- We provide round the clock customer support

- Convenient online submission

- Thorough peer review

- Inclusion in PubMed and all major indexing services

- Maximum visibility for your research

Submit your manuscript at www.biomedcentral.com/submit 